\title{
Highly Efficient Conversion of Solar Energy by the Photoelectric Converter and a Thermoelectric Converter
}

\author{
A. M. Kasimakhunova ${ }^{1}$, Sh. A. Olimov ${ }^{2}$, R. Nurdinova3 ${ }^{3}$, Tahir Iqbal2,4, L. K. Mamadalieva1 \\ ${ }^{1}$ Department of Power Engineering, Fergana Polytechnic Institute, Fergana, Uzbekistan \\ ${ }^{2}$ School of Renewable energy, North China Electric Power University, Beijing, China \\ ${ }^{3}$ Department of Telecommunications Technologies, Tashkent University of Information technologies, Fergana, Uzbekistan \\ ${ }^{4}$ Faculty of Agricultural Engineering \& Technology, PMAS-Arid Agriculture University, Rawalpindi, Pakistan \\ Email: *kasimahunova@rambler.ru
}

How to cite this paper: Kasimakhunova, A.M., Olimov, Sh.A., Nurdinova, R., Iqbal, T. and Mamadalieva, L.K. (2018) Highly Efficient Conversion of Solar Energy by the Photoelectric Converter and a Thermoelectric Converter. Journal of Applied Mathematics and Physics, 6, 520-529.

https://doi.org/10.4236/jamp.2018.63047

Received: December 9, 2017

Accepted: March 18, 2018

Published: March 21, 2018

Copyright (C) 2018 by authors and Scientific Research Publishing Inc. This work is licensed under the Creative Commons Attribution International License (CC BY 4.0).

http://creativecommons.org/licenses/by/4.0/

\begin{abstract}
The analysis of the principle operation of the solar element is given in the work. The efficiency of the creation of combined high-efficiency converters of network energy in electric and thermal is indicated. A method for creating a combined photo thermo converter with a high value of the efficiency of a solar element has been found. A method for separating light from optical lenses is shown. The calculation of the light intensity into diffraction pattern is calculated using the Huygens-Fresnel principle. A new design of a highly efficient combined light-thermal converter into an electrical, with a solar element, operating on selective photoactive radiation is presented. The process of conversion of non-active radiation into electrical radiation by means of a thermo electronic converter is described. The ways of the solution of the problem connected with the reduction of the coefficient of its full effect as a function of temperature and characteristics are indicated.
\end{abstract}

\section{Keywords}

Photo Converter, Spectrum, Electrical Energy, Thermo Converter, Photo-Thermo Converter

\section{Introduction}

Despite many years of research, the issue of increasing the efficiency of conversion of solar radiation into electrical radiation using semiconductor photo converters (PC) remains relevant. In this regard, many works have been done and the results of which are regularly published in periodical scientific and scientific 
and technical journals. A large number of works are devoted to improve the technology of manufacturing solar cells (SC), a number of works notify about the introduced impurities in the structure of the initial materials [1] [2] [3] [4], and there are certain positive results on combining solar cells with thermoelectric converters (TEC) [5] [6]. In addition, there are many publications on the creation of heterojunction and cascade (SC)s; it is impossible to enumerate everything.

Of course, the results of these studies have a noticeable positive outcome. However, because of the main reason, the meaning of which lies in the strong spectral dependence of the conversion coefficient on the spectral composition of the incident radiation, so far, the problem has not been completely solved. Along with the spectral dependence of the efficiency of solar cells, there is the problem of temperature dependence. Although the latter is solved by adding additional coolers to the design of solar converters, all the same, eliminating the cumbersomeness of solar energy sources is not only a weight problem but also economically impractical.

In this regard, the present work is devoted to solve these two latter problems. In our opinion, the creation and introduction of a photo thermoelectric converter (PTEC) with a separate load and selected radiation, gives a very positive result. Conditionally, such a converter is called a photo thermo converter of selective radiation (PTCSI) [7] [8].

The purpose of creating the PTCSI is to find ways to convert only the photoactive part of the light radiation to a photoelectric converter, and the rest of the incident radiation from a thermoelectric converter. Moreover, in this case, since all light radiation with the corresponding spectral composition of light contributes to the creation of an electron-hole pair, there is nothing to heat the PC volume and, consequently, there will be no heating. For TEC, the composition of the light spectrum does not actually play any role. It absorbs all light energy from the side of the light-absorbing surface. This can be achieved by painting the TEC surface with black paint. Further, due to the Seebeck effect, thermal energy is converted into electrical energy.

This research was conducted in Fergana Polytechnic Institute, Fergana, Uzbekistan.

\section{A Short Theory of Light Conversion into Electricity and the Calculation of the Basic Parameters of a Photo Converter}

The working principle of photo converter is the process of interaction of sunlight with a semiconductor crystal during process photons release electrons-carriers of electric charge in crystals. Specially created regions in the volume of crystals with a strong electric field (for example, the so-called pn transitions) trap the arising electrons and separate them in such a way that a current flow in the circuit of the device, and electric power is released on the payload.

According to the principle of operation, the existing types of photo converter 
are divided into two classes: photo elements based on the external photoelectric effect (vacuum and gas-filled), and semiconductor PC with a blocking layer. The same photocells are called gate valves.

Valve photocells favorably differ from all types of photovoltaic devices. This difference lies in the fact that under the influence of light they produce their own electromotive force (EMF). The value of this parameter in several cases in direct sunlight reaches up to one volt. Creating under the action of radiation, its own emf valve photocells, thus directly transferring radiant energy to electrical energy. The occurrence of a gate emf is observed in the case when light generates charge carriers near the potential barrier that is when the metal-semiconductor contacts and p-n junctions are illuminated. Figure 1 shows the photocell switching circuit. The photocell considered in this case as a converter of light energy into electrical energy.

The physical reason for the occurrence of a vented photo-emf is that the potential barrier of the p-n-junction separates the fluxes of photo-holes and photoelectrons. Figure 1 shows the absorption of radiation by a crystal face parallel to the PN junction plane. For assurance, we shall assume that the N-region is illuminated. In the first case, let us consider an open photocell. Electrons and holes created by illumination will diffuse deep into the crystal, and some of their
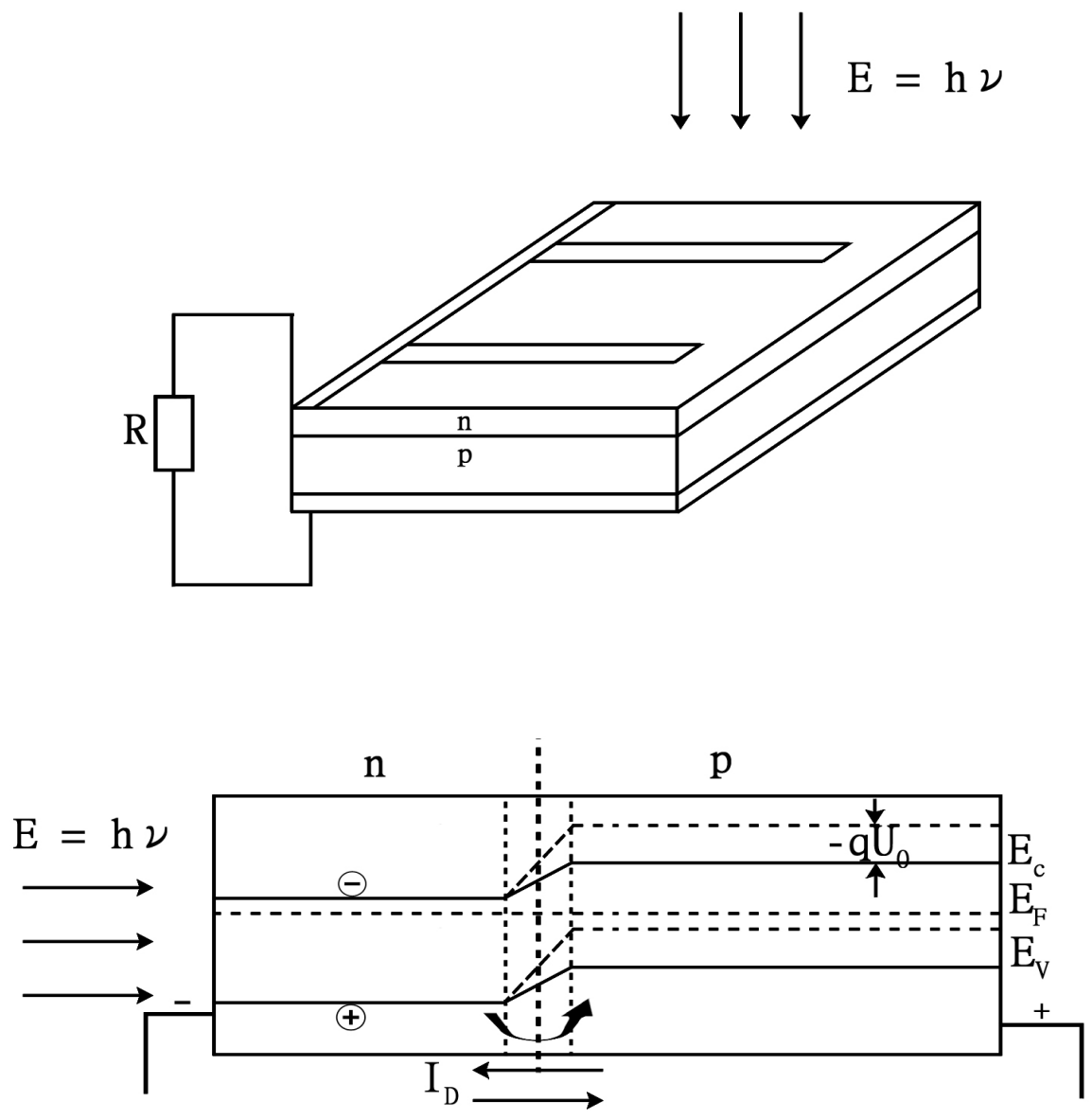

Figure 1. (a) The general view of photo element (b) and its zoned diagram. 
fraction, not having time to recombine (on the surface and in the volume), reaches the PN-junction. Here, the effective work of the photocell is expected when all the pairs created by light reach the contact layer. However, for the main carriers of electrons in the $\mathrm{PN}$ junction there is a potential barrier, and therefore practically all of them will fall in Figure 1 scheme of the decomposition of light radiation into the spectrum.

Here, efficient operation of the photocell is expected when all light by the pair made contact layer. However, for the main carriers of electrons in the p-n-transition there is a potential barrier, and almost all of them fall into the p-region. On the contrary, for the minority carriers, there aren't holes of potential barrier and all reaching the transition of holes are tightened field of transition into the crystal, creating a current I equal in magnitude

$$
I=q S \beta
$$

where $S$-the surface temp of steam generation, $\beta$-share of photo holes of minority carriers which have reached the transition without recombination, $F$-lighted area of the photocell.

The emergence of the current I when photocell light surface results in a negatively charged n-region. p-region, in turn, is positively charged. Consequently, the potential difference arises among the element electrodes. This difference of potentials leads to appear a current Ід due to the injection of holes into the n-region and electrons in p-region. When there isn't recombination in the p-n-transition and currents are small, and if you do not take into account the voltage drops in the thickness of the crystal, then the current can be described as follows:

$$
I_{\text {д }}=I_{s}(\exp (q U / k T)-1)
$$

where $I_{s}=\frac{q D_{p}}{L_{\sigma}}+\frac{q D_{n} n_{p}}{L_{n}}$-saturation current; $q$-electron charge; $k$-is Boltzmann constant; $T$-temperature of the solar cell.

As a result of these currents among the open electrodes of solar cell, the voltage $\mathrm{U} 0$ is established in which the total current $I_{n}=I-I_{q}=0$. When circuit electrodes to an external load voltage decrease among them and currents, $I$ и $I_{q}$ will no longer compensate each other. As a result of it, current flows in a circuit:

$$
I_{n}=I-I_{q}=I-I_{s}(\exp (q U / k T)-1)
$$

Equation (2) is the main gate to the theory of solar cells.

Basic parameters characterizing the solar cell, such as the integral sensitivity and spectral sensitivity is determined not only by its properties but also limits of its application in a particular area. The integral sensitivity of photocell $K$ is the magnitude of photocurrent that flows in a short circuit of the photocell falling on radiant energy flux units consisting of various wavelengths and of their respective spectral content of sunlight radiation. This parameter is given by:

$$
K=\frac{I_{\phi}}{\Phi}
$$


At low light, the photocurrent flows and short-circuit current $I_{\mathrm{к} 3}$ depends on the luminous flux. $\mathrm{C}$ with increasing $\mathrm{F}$, regardless of inclusion in the photoelectric converter external load, this linearity remains. Spectral sensitivity $Q_{\lambda}$ characterizes the magnitude of the photocurrent on the action unit of radiant flux of a certain wavelength. In other words, to determine the spectral sensitivity of the solar cell is taken the attitude short-circuit photocurrent $I_{\mathrm{\kappa} 3}^{\phi}$ to the incident on the photocells flow monochromatic radiation:

$$
Q_{\lambda}=\frac{I_{\mathrm{\kappa} 3}^{\phi}}{\Phi_{\lambda}}
$$

In all cases, the preparation of photovoltaic cells to work when you need to have all the optical data on hand, the value of the spectral sensitivity to any particular wavelength is not enough. Therefore, characteristics of solar cells which are the sensitivity distribution of the spectrum are most commonly used. Therefore, the distribution of the spectral sensitivity in the spectrum gives chances to determine and calculate the current, which flows in the circuit when the photocell is incident on it light flux from any source.

\section{Technique and Method of Separating the Luminous Flux}

Calculation of the light intensity distribution in the diffraction pattern can be performed by the Huygens-Fresnel principle. On the base of the distribution of the radiation in the range of the device, there is a plurality of lenses and prisms can be seen in Figure 2. The action of the prism is based on the dispersion phenomenon that is depending on the refractive index $\varphi$ substance on the light wavelength $\lambda$. The test radiation which is exposed to the allocation of the spectrum, the $S$ passing through the gap, gets on the lens $\pi_{1}$. Slit $S$ on which light is incident, located in the focal $\pi_{1}$ plane of the lens.

This part of the device is called a collimator. The effluent from the lens a parallel beam of light is incident on the prism P. Because of the dispersion, the light of different wavelengths emerges from the prism at different angles. In the focal plane of the lens $\Omega_{2}$ there is a screen or photographic plate, on which the radiation is focused. As a result, the entrance slit image $S$ in the light of different wavelengths appears in different locations of the screen.

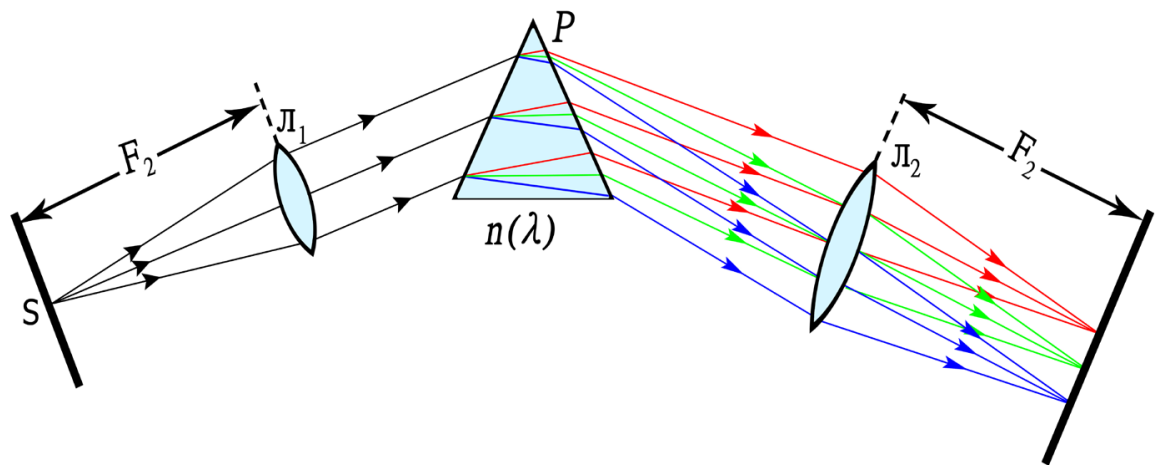

Figure 2. Scheme of location of the light radiation in the spectrum. 
In all solid things (glass, quartz) which are made of the prism, the refractive index $\mathrm{n}$ in the visible light range decreases with increasing wavelength $\lambda$, so the prism deflects most strongly from the original direction of blue and violet light and least-red. Modern technology allows splitting light fluxes from the spectra without much difficulty. This can be successfully applied high spectral class of devices consisting of a diffraction grating

To observe interference maximum, path difference $\Delta$ among the waves emitted by the adjacent slots on the screen which focuses the radiation should be equal to the whole number of wavelengths:

$$
\Delta=d \sin \Theta_{m}=m \lambda
$$

Here $d$-grating period, $m=0, \pm 1, \pm 2, \cdots$-the order of the diffraction peak. In the focal plane of the lens distance ym from the zero order maximum $(m=0)$ to a maximum of $\mathrm{m}$-th order at small angles of the diffraction is equal:

$$
y_{m}=m \frac{\lambda}{\alpha} F,
$$

where $F$-focal distance.

It should be noted that $N$ interference waves arriving at the lattice point $N$ of slits occurs in each point of the focal plane of the lens. This is explained by a lot of the wave (or "multi-beam") interference. In the transition from the main peak to the neighboring minimum path difference $\Delta=d \sin \theta$ should be changed to $\lambda / N$. From this condition the angular half-width $\delta \theta$ of major peaks can be estimated:

$$
\Delta \delta=\delta(d \sin \Theta)=d \cos \Theta \delta \Theta \approx d * \delta \Theta=\frac{\lambda}{N}
$$

Here, for simplicity, it is assumed that the diffraction angles are quite small. Consequently,

$$
\delta \Theta=\frac{\lambda}{N d}
$$

where $N d$-full size of bars.

This ratio is in complete agreement with the theory of diffraction in parallel rays, according to which the diffraction divergence of the parallel beam is the ratio of the wavelength $\lambda$ to the transverse dimension of the obstacles. Here we can draw an important conclusion that diffraction of light on the grid the main peaks are extremely narrow. Figure 3 gives an idea about how to change the sharpness of the main peaks with an increase in the number of grating slits. From the diffraction grating, the position of the major peaks depends on the wavelength $\lambda$. Therefore, the lattice is able to decompose the light into a spectrum, that is, it is a spectral device.

The technique of designing and using a highly efficient combined converter for more detailed information on existing photo thermoelectric transducers, one can familiarize with the previous works of these somewhere else [6] [9]. This development is initially just like a simple photocell, contributed to the overall ef- 
ficiency, substantially, but not too much. The results of a study on the design of photo thermo transducers (PTC) of a more perfect design led to the production of a sample as shown in Figure 4, in which two important problems can be solved: an increase in the value of the electric efficiency transformation and elimination of temperature deterioration of electrophysical parameters of semiconductor materials.
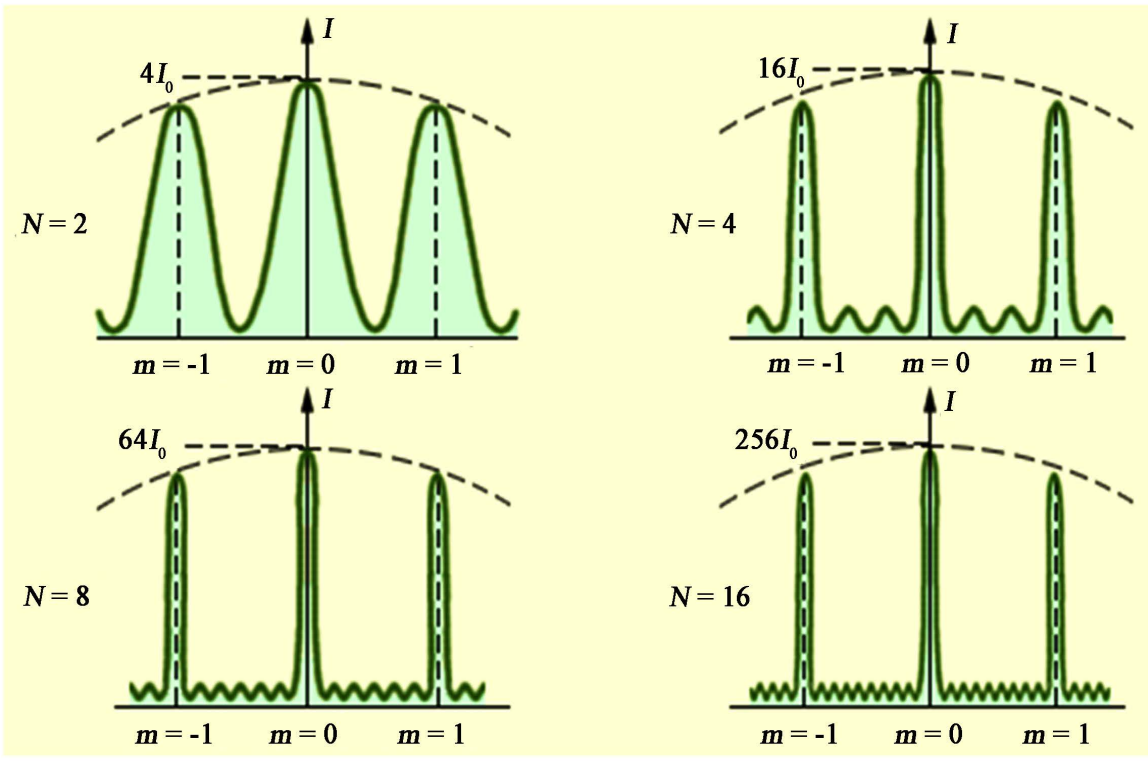

Figure 3. Distribution of intensity in the diffraction of monochromatic light on lattices with a different number of slots. $I_{0}$-intensity vibrations at light diffraction on one slit.

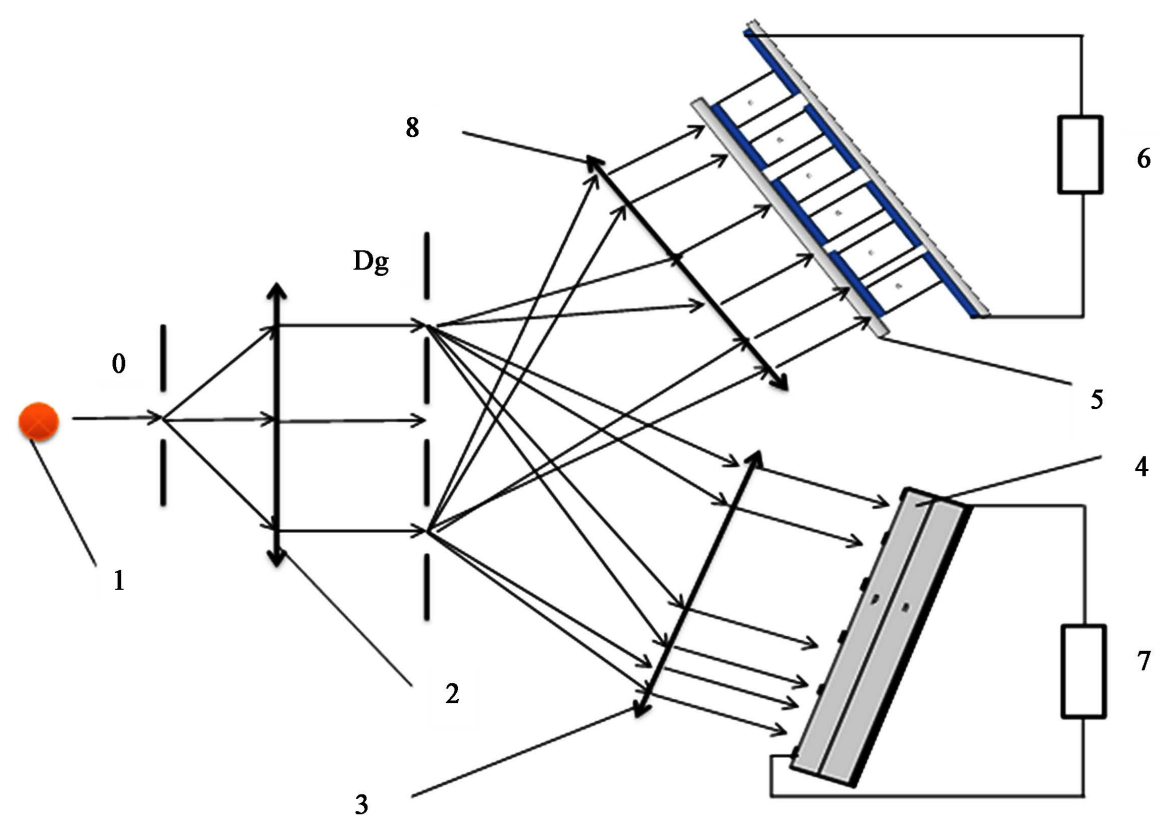

Figure 4. The scheme of distribution of light radiation on the surface of photoelectric and thermoelectric converters. 1-light source, 2, 3, 8-set of optical glasses, 4-photo converter, the 5-front surface of thermo converter, 6-load of thermo converter, 7-load of photo converter, 0 - enter hole, Dg-diffraction grating. 
This is achieved in the construction of a PTC, in which the photo- and thermoelectric parts of the transducers are exposed to illumination with light with different separated spectral characteristics. After the overheating of the photoelectric converter took place due to not the photo of the active part of the solar radiation. The light passing into the PEC volume does not completely turn into an electric one. Part of the radiation, being absorbed in the volume, turns into heat. The fact that the rise in the temperature of the photoconductivity has a negative effect on the efficiency of the transformation has been known for a long time. There are many works devoted to the temperature dependences of the electrophysical parameters of semiconductors.

There is a lot of work devoted to the temperature dependences of the electrical parameters of semiconductors. Thus, the photo thermo-transformer with the light scattered in the spectrum (shown in Figure 4) is illuminated so that the radiation producing the electron-hole pairs falls on the frontal surface of the photoelectric converter. This is achieved using a set of lenses and prisms 2 and 3 , working on the principle of Huygens-Fresnel. A set of optical filters, repeatedly refracting radiation, distributes it into two parts. Basic-the first part of the radiation mainly consists of short-wave and partially visible spectral regions. It is directed to the frontal surface of the photo converter 4 . Another-a longer wavelength ("warm") radiation, entering the upper hot commutation plates 5 of the thermo converter (TC) is converted into an electrical one. As is known, for TIC, the spectral composition of light does not have a significant significance. It's important for him to become heat. The thermal energy of the hot junction contributes to the generation of electricity in addition to the electric power of the photoelectric method of pre-formation.

The above variant of the photo thermo-transformer working on especially distributed light is switched on to separate loads. The load of the PEC is 7 and the load of TEC-6. However, the theoretical calculation shows that they can be included in the total load. For this, it is necessary to select the number and geometric dimensions of the branches of the thermo element, and the number of TEs.

The advantage of the present design of selective transducers is that in this case heating of photoelectric converters disappears. Consequently, it is possible to maintain practically the values of the efficiency of the PEC at the highest light intensities. And the formula

$$
\eta_{F E P}=\frac{W_{\text {us }}^{\text {max.FEP }}}{W_{\text {fall. }}^{1}}
$$

shows that this parameter becomes substantially higher than before, that is, the same (PC) without any constructive changes gives a greater value of $\eta_{P E}$. This is explained by the following: in Formula (9), the expression in the denominator, in contrast to traditional calculations,

$$
\eta_{F E P}=\frac{W_{u s}^{\max . F E P}}{W_{\text {fall. }}}
$$


This is explained as followings: in the formula (9) the expression in the denominator, unlike traditional computing, (10) is a quantity equal to $W_{\text {fall. }}^{1}=(1-k) W_{\text {fall. }}-Q$, where $-W_{u s}^{\text {max.FEP }}$ the maximum usable electric power generated by solar cells on the load-photoactive part of the light radiation coming to the surface of the solar cells, $W_{\text {fall }}$-integrated directional light output from the light source to foto termo converter, $k$-the coefficient of reflection of light from the front surface of the photo converter. For the calculation was taken to be $20 \%$.

Accordingly, efficiency $=$ thermo converter

$$
\eta_{\text {TEC }}=\frac{W_{\text {us }}^{\text {max.TEC }}}{(1-k) W_{\text {fall }}-W_{\text {fall }}^{1}}=\frac{W_{u s}^{\text {max.TEC }}}{Q_{T E C}} ;
$$

In the last formula a useful electric power generated by the load thermo converter, while covering its front surface. $Q_{T E C}=W_{\text {fall }}^{\prime \prime}$ light power passing on the ceramic plate on the surface of the thermo converter.

It is the actual heat flow that will be sent to the hot junctions of the thermo electrical converter as a front surface of the thermo electrical converter painted on the black color, which corresponds to one hundred percent of the absorption coefficient of a black body.

\section{Conclusions}

In conclusion, it should be noted that the proposed method of combining and converting light radiation gives high values of efficiency, without significant economic and constructive costs. In addition, it is an original way of eliminating the parasitic heat effect in the volume of the (PC). To find ways to improve the efficiency of the photoelectric method of converting light to electricity, there are still prospects for the development of research and design work, we can assume that there is a way to recycle not the photo of active light from photoelectric conversion using solar collectors. Modern solar collectors, when finding the optimal design option, can work quite successfully in combination with (PC). And in this case, there is a gain-it is saving energy of heat, which is not an unimportant factor in our time!

Notice!

Photo-thermo converter of selective radiation recommended by us doesn't have analogs.

\section{References}

[1] Lidorenko, D.S., Evdokimov, N.S., Zaitseva, V.M., Koltun, A.K., Riabikov, M.M. and Strebkov, S.V. (1978) New Models of Solar Cells and Prospects for Their Optimization. Applied Solar Energy, 14, 1-12.

[2] Lindmayer, J. and Allison, J.F. (1990) The Violet Cell: An Improved Silicon Solar Cell. Solar Cells, 29, 151-166. https://doi.org/10.1016/0379-6787(90)90023-X

[3] Project, I. (2017) Solar Energy Conversion Systems' Monograph. http://www.istc.int/en/project/F7EC83CB7756208BC3256C79002C556B 
[4] Peterson, G., Su, Q., Wang, Y., Dowben, P.A. and Nastasi, M. (2015) Improved p-n Heterojunction Device Performance Induced by Irradiation in Amorphous Boron Carbide Films. Materials Science and Engineering. B, 202, 25-30. https://doi.org/10.1016/j.mseb.2015.09.002

[5] Iordanishvili, E.K. (2006) Photothermoelectric Converter: A New Design Concept. Technical Physics Letters, 32, 1077-1078. https://doi.org/10.1134/S1063785006120248

[6] Kasymahunova, A.M. (2003) Fotothermoelectrical Converters and Their Application. Monograph, 2003.

[7] Bashmakov, Y.A. (2015) Cherenkov Radiation: From Discovery to RICH. Physics-Uspekhi, 58, 467-471. https://doi.org/10.3367/UFNe.0185.201505f.0502

[8] Landsberg, G.S. (1976) Optica, Moscow. Science, 1976, 198-205-215.

[9] Borisov, T.P.D.S.N., Gorodetsky, S.M., Iordanishvili, E.K., Lyubashevskaya, T.L., Kagan, M.V. and Kasymakhunov, A.M. (1980) The Study of Energy Characteristics Fototermoelements. "FAN" Solar Technology. 